\section{Interclinician disparity in periodontal decision making: need for consensus statements on surgical treatment}

Cosyn J, De Bruyn H. Interclinician disparity in periodontal decision making: need for consensus statements on surgical treatment. J Periodont Res 2007; 42: 311-317. (C) 2007 The Authors. Journal compilation (C) 2007 Blackwell Munksgaard

Background and Objective: The clinical factors involved in the decision-making process for surgical treatment have been described. However, there is still little standardization of the criteria upon which such a decision should be based. The impact of this lack of practice guidelines on the recommendation of surgery in clinical practice is unclear. The objective of the present study was to investigate the recommendation of surgical therapy during the corrective/reparative treatment phase by trained clinicians with various backgrounds on the basis of clinical data.

Material and Methods: Fifteen clinicians (10 periodontal students and/or recent graduates from two dental schools and five experienced practitioners) were asked to make a treatment decision (surgery or no surgery) at a tooth level. Therefore, they were given 23 initially treated patients with details on demographics and smoking habits. Radiographs and clinical information on 573 teeth at baseline (prior to root debridement) and at 9 mo of follow-up were provided.

Results: Clinicians interpret clinical data quite differently in their advice of surgery when practice guidelines are not provided, as the results showed high variation in surgical recommendation. Experienced practitioners showed most variation, with a range from 13 to $50 \%$ in surgical recommendation. Clinicians linked to a training center shared a common treatment philosophy as to when periodontal surgery should be performed. This philosophy differed markedly among the two dental schools. Most disagreement among the 15 clinicians was found for deep pockets and for multirooted teeth. Disease status, tooth type, age, and full-mouth plaque levels had a significant impact on decision making.

Conclusion: The substantial variation in recommending surgery calls for consensus statements on surgical treatment.
J. Cosyn 1, H. De Bruyn²

${ }^{1}$ Free University of Brussels (VUB), School

of Dental Medicine, Department of

Periodontology, Brussels, Belgium and

${ }^{2}$ Universtity of Ghent, School of Dental Medicine,

Department of Periodontology, Ghent, Belgium
Dr Jan Cosyn, Department of Periodontology, School of Dental Medicine, Free University of Brussels (VUB), Laarbeeklaan 103, B-1090 Brussels, Belgium

Tel: +3224774960 or +3249610012

Fax: +3224774902

e-mail: jan.cosyn@vub.ac.be

Key words: decision making; periodontal surgery; periodontitis; scaling and root planing

Accepted for publication August 21, 2006
Decision making is an important aspect of daily clinical practice. Defining the objectives of therapy is a crucial step in this process. The objectives of therapy are essentially driven by the patient's perceived problems and demands, the patient's profile, and technical factors related to the care provider (experience, skills, etc.) and receiver (access, visibility, anatomic limitations, etc.). The patient's profile may be defined as the sum of all behavioural and biologic determinants that characterize an individual and serve to evaluate that individual's aptitude to undergo active therapy (1). Hitherto, decision-making analyses in reference to periodontal treatment have mainly focused on biologic determinants. These include factors involved in periodontal risk assessment. 
After all, risk indicators, factors, and determinants for periodontitis not only increase the probablility of developing disease, they are also critical in its prevention and treatment (1). Other factors influencing periodontal treatment decisions comprise clinical parameters - probing pocket depth, clinical attachment level, furcation involvement, and tooth mobility are highly associated with the recommendation to perform periodontal surgery (2). This is logical, as ample metaanalyses have shown the therapeutic significance of the degree of periodontal destruction. Periodontal surgery may result in significantly greater pocket reduction and clinical attachment gain than scaling and root planing at deep sites ( $\geq 7 \mathrm{~mm}$ probing pocket depth) (3-6). In the treatment of medium-to-deep pockets (4-6 mm probing pocket depth), open flap debridement results in equal to slightly greater pocket reduction than scaling and root planing. However, the opposite is true considering clinical attachment level as the response parameter (3-5). When periodontal therapy is conducted in shallow pockets ( $\leq 3 \mathrm{~mm}$ probing pocket depth), deterioration in clinical attachment level may be expected, especially following flap elevation $(5,7)$. These evidence-based guidelines have simpli- fied the choice between surgical and nonsurgical periodontal therapy. In addition, one should keep in mind the fact that their objectives are different: scaling and root planing are conducted during the hygienic phase in order to eliminate local and systemic etiologic factors, hereby re-establishing a healthcompatible microflora, whereas surgery is performed during the corrective/reparative phase for which elimination of local defects and regeneration of lost tissues are the ultimate goal. As described above, treatment objectives are fundamental in the decision-making process.

An important prerequisite for successful therapy is adequate plaque control and supportive care (8-10). In particular, when periodontal surgery is planned, plaque control is a pivotal factor. That is, marked pocket reduction and clinical attachment gain can be expected following surgery at deep sites if an intensive postoperative oral hygiene regimen is provided (11). However, when plaque levels are high, probing pocket depth tends to relapse, and clinical attachment loss of $\approx 1 \mathrm{~mm}$ after 1 year is to be expected following surgical treatment (8). This observation is detrimental, knowing that in the absence of therapy only $0.3 \mathrm{~mm}$ clinical attachment loss annually occurs $(9,10,12)$. These findings suggest that

Table 1. Clinician's background

\begin{tabular}{llll}
\hline Clinician & Periodontal training & Gender & $\begin{array}{l}\text { Age } \\
\text { (years) }\end{array}$ \\
\hline P1 & Catholic University of Leuven (KUL) & M & 49 \\
P2 & Université de Paris (Paris 7) & F & 48 \\
P3 & Université Catholic de Louvain (UCL) & M & 50 \\
P4 & University of Ghent (UGent) & F & 50 \\
P5 & Free University of Brussels (VUB) & M & 43 \\
G1 & University of Ghent (UGent) & F & 26 \\
G2 & University of Ghent (UGent) & M & 30 \\
G3 & University of Ghent (UGent) & F & 26 \\
G4 & University of Ghent (UGent) & M & 29 \\
G5 & University of Ghent (UGent) & F & 34 \\
B1 & Free University of Brussels (VUB) & M & 28 \\
B2 & Free University of Brussels (VUB) & M & 29 \\
B3 & Free University of Brussels (VUB) & M & 39 \\
B4 & Free University of Brussels (VUB) & F & 27 \\
B5 & Free University of Brussels (VUB) & F & 29
\end{tabular}

$\mathrm{B}$, clinician connected to the dental school of the University of Brussels with fewer than 5 years of experience in periodontology; $\mathrm{G}$, clinician connected to the dental school of the University of Ghent with fewer than 5 years of experience in periodontology; $\mathrm{P}$, clinician in private practice with at least 15 years of experience in periodontology.

$\mathrm{F}$, female; $\mathrm{M}$, male. periodontal surgery should be avoided in noncompliant patients.

Even though the clinical factors involved in the decision-making process for surgical treatment have been described, the management of this information in the advice of surgery by the clinician remains unclear. The objective of the present study was to investigate the recommendation of surgical therapy during the corrective/ reparative treatment phase by trained clinicians from various backgrounds.

\section{Material and methods}

\section{Clinicians}

Fifteen trained clinicians were asked to make a surgical treatment decision, irrespective of the technique, at a tooth level. Therefore, they were given 23 initially treated patients. No specific guidelines in reference to clinical practice were discussed prior to decisionmaking, and all clinicians scored independently. Table 1 gives an overview of the clinician's background. Ten clinicians had fewer than 5 years of experience in periodontology. They had recently graduated or were still in training. Of these 10 clinicians, five were associated with the dental school of the Free University of Brussels and five were associated with the dental school of the University of Ghent. The remaining five specialists had at least 15 years of private clinical experience in periodontology, and all had undergone their original training at a different dental school. The training programs of each of the 15 clinicians consisted of 3 years of full-time education in periodontology (including theoretical, clinical and research training). In Belgium, this type of training is considered a prerequisite to become officially recognized as a dental specialist in periodontology. To date, only the graduate program in periodontology of the Catholic University of Leuven has been internationally accredited by the European Federation of Periodontology.

\section{Subjects}

The 23 subjects comprised systemically healthy patients (eight men and 15 
women) with a mean age of $50 \pm 13$ years. Four were smokers ( $\geq 10$ cigarettes a day). All patients had at least 20 teeth (median: 25) with a minimum of four multirooted teeth (median: $n=6$ ). Considering the shortened dental arch concept as a minimal objective for subjects of at least 50 years old, there were no prosthetic treatment needs for any of the 23 subjects. However, 33 teeth in 16 patients required restorative treatment by means of crowns.

The initial debridement phase had been completed in all 23 patients 9 mo prior to the periodontal evaluation. Debridement consisted of ultrasonic and manual scaling and root planing, conducted by one and the same experienced clinician in two sessions with a time interval of $1 \mathrm{wk}$ between both treatment sessions. At 1, 3, and 6 mo of follow-up, oral hygiene had been reinforced and supragingival cleaning had been performed. All clinical parameters at baseline and at 9 mo of follow-up had been recorded by an experienced clinician who had been calibrated, prior to baseline recordings and on the basis of duplicate measurements $(n=414)$, for the plaque index (13), probing pocket depth, and clinical attachment level in three patients with chronic periodontitis. Intra-examiner repeatability was good to excellent for the plaque index (Spearman's correlation: $r=$ $0.86 ; \quad p<0.001)$, probing pocket depth (Pearson's correlation: $r=0.92$; $p<0.001)$, and clinical attachment level (Pearson's correlation: $r=0.91$; $p<0.001)$. Table 2 summarizes the degree of periodontal destruction at baseline and the clinical response to scaling and root planing at 9 mo of follow-up.

In order to make surgical treatment decisions for 573 teeth, all clinicians had access to demographic details, smoking habits, intra-oral long-cone radiographs, and all clinical information from baseline (prior to therapy) and at 9 mo of follow-up comprising data on probing pocket depth, clinical attachment level, furcation involvement, tooth mobility, bleeding on probing, sulcus bleeding index (14), and plaque index per tooth.

\section{Statistical analysis}

Data analysis was performed using the tooth as the experimental unit. All treatment decisions made by the 15 clinicians at a tooth level were dichotomized ( $0=$ no surgery; $1=$ surgery). Subsequently, pairwise comparisons were conducted between the 15 clinicians. Percentage identical scores and kappa statistics were adopted to describe the level of agreement between clinicians.

The impact of the disease status (residual probing pocket depth and loss of clinical attachment level at 9 mo of follow-up) on the recommendation of surgical treatment was evaluated by means of one-way analysis of variance. If a statistically significant difference was observed, the Scheffe's test was performed to determine its source. In order to identify other influencing factors on the recommendation of surgery, the general linear model approach was used to perform analysis of covariance with the deepest residual probing pocket depth per tooth as a covariate, hereby controlling for disease status. The level of significance was set at $5 \%$.

\section{Results}

\section{Interclinician's agreement}

For each clinician, the level of agreement with other clinicians is summarized in Table 3. Overall, P1 showed the largest dissimilarity in scoring with the other clinicians, as the median percentage agreement $(64 \%)$ and kappa score (0.27) were very low. Most disagreement was found between $\mathrm{P} 1$ and B4, with only $54 \%$ agreement, corresponding to a kappa score of 0.09 .

Table 3 shows the level of agreement for each of the practitioners with clinicians from a different group and with clinicians from the same group. Apart from experienced practitioners in private practice, agreement scores were systematically higher among clinicians from the same group. This observation was most obvious for clinicians from the dental school in Brussels, indicating good to excellent agreement among them (agreement ranging from 77 to $93 \%$; kappa scores ranging from 0.40 to 0.70 ). In constrast, lower agreement between these clinicians and practitioners from the other groups was found (agreement ranging from 54 to $95 \%$; kappa scores ranging from 0.09 to 0.74 ).

\section{Treatment decisions}

Table 4 shows the frequency of recommending periodontal surgery per practitioner. Surgical therapy was recommended for $13-50 \%$ of all teeth by the specialists with at least 15 years of clinical experience. Practitioners connected to the dental school of Ghent advised surgery for $25-28 \%$ of all teeth. Clinicians connected to the dental school in Brussels selected periodontal surgery as the treatment of choice for only $6-15 \%$ of the same experimental teeth.

Besides these quantitative disparities, the following qualitative aspects need to be considered: of all the teeth for which surgery was advised, at least $63 \%$ were multirooted teeth and at least $58 \%$ were teeth with deep residual pockets, as recommended by clinicians

Table 2. Degree of periodontal destruction at baseline and clinical effects of therapy at 9 mo of follow-up (mean \pm SD)

\begin{tabular}{|c|c|c|c|c|c|c|c|}
\hline & SBI & PI & BoP $(\%)$ & $\begin{array}{l}\text { Full-mouth } \\
\text { PPD }\end{array}$ & $\begin{array}{l}\text { Percentage of } \\
\text { pockets } 0-3 \mathrm{~mm} \text { PPD }\end{array}$ & $\begin{array}{l}\text { Percentage of } \\
\text { pockets } 4-6 \mathrm{~mm} \text { PPD }\end{array}$ & $\begin{array}{l}\text { Percentage of pockets } \\
\geq 7 \mathrm{~mm} \text { PPD }\end{array}$ \\
\hline Baseline & $1.24 \pm 0.61$ & $2.53 \pm 0.53$ & $73 \pm 14$ & $4.84 \pm 0.56$ & $31 \pm 12$ & $47 \pm 10$ & $22 \pm 8$ \\
\hline $\begin{array}{l}9 \text { mo of } \\
\text { follow-up }\end{array}$ & $0.35 \pm 0.17$ & $1.75 \pm 0.47$ & $24 \pm 6$ & $3.30 \pm 0.51$ & $69 \pm 14$ & $28 \pm 11$ & $3 \pm 4$ \\
\hline
\end{tabular}

BoP, bleeding on probing; PI, plaque index; PPD, probing pocket depth; SBI, sulcus bleeding index. 
Table 3. Interclinician's agreement

\begin{tabular}{|c|c|c|c|c|c|c|}
\hline \multirow[b]{2}{*}{ Clinician } & \multicolumn{2}{|c|}{ Agreement with other clinicians ${ }^{\mathrm{a}}$} & \multicolumn{2}{|c|}{$\begin{array}{l}\text { Agreement with other clinicians } \\
\text { from a different group }{ }^{\mathrm{a}}\end{array}$} & \multicolumn{2}{|c|}{$\begin{array}{l}\text { Agreement with other clinicians } \\
\text { from the same group }\end{array}$} \\
\hline & $\%$ & Kappa & $\%$ & Kappa & $\%$ & Kappa \\
\hline P1 & $64(54 ; 71)$ & $0.27(0.09 ; 0.42)$ & $63(54 ; 71)$ & $0.25(0.09 ; 0.42)$ & $66(58 ; 69)$ & $0.32(0.16 ; 0.38)$ \\
\hline $\mathrm{P} 2$ & $86(58 ; 95)$ & $0.47(0.16 ; 0.74)$ & $88(78 ; 95)$ & $0.51(0.34 ; 0.74)$ & $76(58 ; 87)$ & $0.28(0.16 ; 0.46)$ \\
\hline P3 & $78(68 ; 82)$ & $0.35(0.14 ; 0.56)$ & $77(73 ; 80)$ & $0.33(0.14 ; 0.47)$ & $78(68 ; 82)$ & $0.37(0.27 ; 0.56)$ \\
\hline P4 & $75(68 ; 82)$ & $0.37(0.17 ; 0.59)$ & $76(68 ; 82)$ & $0.36(0.17 ; 0.59)$ & $75(69 ; 82)$ & $0.37(0.30 ; 0.56)$ \\
\hline P5 & $84(64 ; 87)$ & $0.39(0.27 ; 0.53)$ & $86(77 ; 86)$ & $0.40(0.27 ; 0.53)$ & $77(64 ; 87)$ & $0.36(0.28 ; 0.46)$ \\
\hline G1 & $83(67 ; 90)$ & $0.50(0.32 ; 0.75)$ & $83(67 ; 87)$ & $0.47(0.32 ; 0.61)$ & $84(76 ; 90)$ & $0.57(0.34 ; 0.75)$ \\
\hline G2 & $79(71 ; 85)$ & $0.41(0.21 ; 0.62)$ & $79(71 ; 80)$ & $0.40(0.21 ; 0.49)$ & $83(74 ; 85)$ & $0.56(0.32 ; 0.62)$ \\
\hline G3 & $83(69 ; 90)$ & $0.50(0.29 ; 0.75)$ & $82(69 ; 87)$ & $0.49(0.29 ; 0.62)$ & $85(75 ; 90)$ & $0.62(0.34 ; 0.75)$ \\
\hline G4 & $77(63 ; 81)$ & $0.31(0.17 ; 0.37)$ & $79(63 ; 81)$ & $0.27(0.17 ; 0.34)$ & $76(74 ; 77)$ & $0.34(0.32 ; 0.37)$ \\
\hline G5 & $83(70 ; 86)$ & $0.46(0.29 ; 0.61)$ & $83(70 ; 86)$ & $0.44(0.29 ; 0.56)$ & $84(77 ; 85)$ & $0.58(0.37 ; 0.61)$ \\
\hline $\mathrm{B} 1$ & $84(56 ; 95)$ & $0.44(0.13 ; 0.74)$ & $82(56 ; 95)$ & $0.35(0.13 ; 0.74)$ & $92(89 ; 93)$ & $0.59(0.46 ; 0.66)$ \\
\hline B2 & $84(60 ; 93)$ & $0.43(0.20 ; 0.70)$ & $82(60 ; 88)$ & $0.39(0.20 ; 0.51)$ & $90(89 ; 93)$ & $0.48(0.40 ; 0.70)$ \\
\hline B3 & $87(62 ; 93)$ & $0.54(0.24 ; 0.70)$ & $83(62 ; 93)$ & $0.46(0.24 ; 0.70)$ & $92(91 ; 93)$ & $0.67(0.52 ; 0.70)$ \\
\hline B4 & $81(54 ; 93)$ & $0.29(0.09 ; 0.54)$ & $79(54 ; 92)$ & $0.24(0.09 ; 0.52)$ & $91(90 ; 93)$ & $0.52(0.40 ; 0.54)$ \\
\hline B5 & $85(57 ; 94)$ & $0.46(0.15 ; 0.71)$ & $81(57 ; 94)$ & $0.40(0.15 ; 0.71)$ & $92(89 ; 91)$ & $0.57(0.50 ; 0.68)$ \\
\hline
\end{tabular}

B, clinician connected to the dental school of the University of Brussels with less than 5 years of experience in periodontology. G, clinician connected to the dental school of the University of Ghent with less than 5 years of experience in periodontology. $\mathrm{P}$, clinician in private practice with at least 15 years of experience in periodontology.

${ }^{a}$ Median percentage agreement and median kappa scores based on pairwise comparisons (minimum; maximum).

Table 4. Treatment decisions

\begin{tabular}{|c|c|c|c|c|c|}
\hline \multirow[b]{2}{*}{ Clinician } & \multirow{2}{*}{$\begin{array}{l}\text { Frequency } \\
\text { of surgical } \\
\text { therapy }(\%)^{\mathrm{a}}\end{array}$} & \multicolumn{2}{|c|}{$\begin{array}{l}\text { Proportional distribution of } \\
\text { surgeries per tooth type }\end{array}$} & \multicolumn{2}{|c|}{$\begin{array}{l}\text { Proportional distribution of } \\
\text { surgeries per residual PPD }\end{array}$} \\
\hline & & $\begin{array}{l}\text { Single-rooted } \\
\text { teeth }(\%)^{\mathrm{b}}\end{array}$ & $\begin{array}{l}\text { Multirooted } \\
\text { teeth }(\%)^{\mathrm{b}}\end{array}$ & $\begin{array}{l}\text { 4-6 mm residual } \\
\operatorname{PPD}(\%)^{\mathrm{c}}\end{array}$ & $\begin{array}{l}\geq 7 \mathrm{~mm} \text { residual } \\
\mathrm{PPD}(\%)^{\mathrm{c}}\end{array}$ \\
\hline P1 & 50 & 67 & 33 & 81 & 19 \\
\hline $\mathrm{P} 2$ & 13 & 32 & 68 & 40 & 60 \\
\hline P3 & 25 & 53 & 47 & 74 & 26 \\
\hline P4 & 33 & 58 & 42 & 75 & 25 \\
\hline P5 & 16 & 55 & 45 & 60 & 40 \\
\hline G1 & 25 & 40 & 60 & 56 & 44 \\
\hline G2 & 28 & 50 & 50 & 67 & 33 \\
\hline G3 & 28 & 45 & 55 & 60 & 40 \\
\hline G4 & 22 & 54 & 46 & 65 & 35 \\
\hline G5 & 25 & 57 & 43 & 61 & 39 \\
\hline B1 & 11 & 33 & 67 & 33 & 67 \\
\hline B2 & 12 & 37 & 63 & 34 & 66 \\
\hline B3 & 15 & 35 & 65 & 31 & 69 \\
\hline B4 & 6 & 22 & 78 & 19 & 81 \\
\hline B5 & 13 & 37 & 63 & 42 & 58 \\
\hline
\end{tabular}

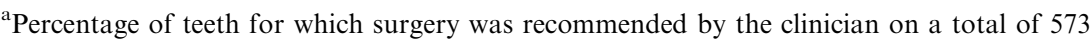
teeth.

${ }^{\mathrm{b}}$ Percentage of single-rooted, respectively, multirooted teeth for which surgery was recommended on the total number of teeth scheduled for surgery.

'Percentage of teeth with medium deep residual pockets, respectively, deep residual pockets for which surgery was recommended on the total number of teeth scheduled for surgery.

PPD, probing pocket depth.

connected to the dental school in Brussels. Except for P2, the other practitioners in private practice and from the dental school in Ghent proportionally advised less surgery for multirooted teeth $(33-60 \%)$ and for teeth with residual deep pockets (1944\%) (Table 3).

When pairwise comparisons were performed between the 15 clinicians, most disagreement was found for deep pockets $(\geq 7 \mathrm{~mm}$ probing pocket depth) and for multirooted teeth (Table 5).

\section{Influencing factors on decision making}

As shown in Table 6, the disease status was strongly related to the overall recommendation of surgical treatment: the higher the residual probing pocket depth and loss of clinical attachment level, the more surgery was advised. In addition, tooth type, age, and fullmouth plaque index had a significant impact on decision making when corrected for disease status. Factors such as restorative treatment need, gender, smoking, and full-mouth bleeding on probing did not have a significant influence on the overall recommendation of surgery.

\section{Discussion}

Decision-making analyses in the different fields of dentistry are emerging. In particular, therapeutic variation by general practitioners, and factors influencing the dental treatment of choice, have been extensively investigated (15-19).

As in other disciplines, decision making in periodontology is complex 
because numerous factors may influence a clinical decision. Essentially, any decision should be driven by the objectives of therapy, the patient's perceived problems and demands, his/ her profile, and technical restrictions (1). When all these determinants have been taken into account and surgical treatment is proposed, acceptance by the patient is not always self-evident: patient's anxiety and trust in the care provider appear critical in this matter (20).

The clinical factors involved in making a surgical treatment decision have been described previously, resulting in a logistic regression model to assist the clinician in making such a decision (2). These clinical factors mainly describe disease severity. The results of this study confirmed a strong, positive link between disease status and the recommendation of periodontal surgery. In addition, tooth type, age and full-mouth plaque index had a significant impact on decision making when corrected for disease status. That is, surgery was more frequently recommended for multirooted teeth, which is not surprising because these teeth may present furcation involvement. Needless to say, maximal access to these loci minori resistentiae can be achieved by means of surgery. Surgical treatment was also more frequently advised in young patients. The observation that practitioners had a tendency to engage a more drastic strategy to eliminate pathology in younger patients seems to be in accordance with clinical experience. Finally, surgery was more frequently recommended for patients with low full-mouth plaque levels. This is also logical knowing that oral hygiene is a pivotal factor in the outcome of periodontal surgery (8-12).

The results of this study, based on clinical data, indicate high variation in the advice of surgical treatment on a tooth-by-tooth basis. Even though the disease status was positively related to the recommendation of surgery, there was more disagreement among the 15 clinicians for deep pockets ( $\geq 7 \mathrm{~mm}$ probing pocket depth) than for medium-to-deep pockets $(4-6 \mathrm{~mm}$ probing pocket depth). This is sur-

Table 5. Clinical characteristics in relation to the level of agreement between 15 clinicians

\begin{tabular}{lrl}
\hline Characteristic & $n$ & Agreement \pm SD $(\%)^{\mathrm{a}}$ \\
\hline Residual PPD & & \\
4-6 mm residual PPD & 319 & $73 \pm 13$ \\
$\geq 7 \mathrm{~mm}$ residual PPD & 65 & $63 \pm 13$ \\
Tooth type & & \\
$\quad$ Single-rooted teeth & 435 & $83 \pm 9$ \\
Multirooted teeth & 138 & $67 \pm 10$ \\
\hline
\end{tabular}

n, number of teeth; PPD, probing pocket depth.

${ }^{\mathrm{a}}$ Mean percentage agreement based on pairwise comparisons between 15 clinicians $\pm \mathrm{SD}$.

Table 6. Influencing factors on the recommendation of surgical treatment by 15 clinicians

\begin{tabular}{|c|c|c|c|}
\hline Factor & $n$ & $\begin{array}{l}\text { Frequency of } \\
\text { surgical } \\
\text { therapy }(\%)^{\mathrm{a}}\end{array}$ & Significant \\
\hline \multicolumn{4}{|l|}{ Disease status } \\
\hline \multicolumn{4}{|l|}{ Residual PPD } \\
\hline$\bullet \geq 3 \mathrm{~mm}$ residual $\mathrm{PPD}$ & 189 & 0 & \multirow{3}{*}{$\begin{array}{l}\text { Yes } \\
(p<0.001)\end{array}$} \\
\hline - 4-6 mm residual PPD & 319 & $23^{\mathrm{c}}$ & \\
\hline$\bullet \geq 7 \mathrm{~mm}$ residual PPD & 65 & $72^{\mathrm{c}}$ & \\
\hline \multicolumn{4}{|l|}{ Residual loss of CAL } \\
\hline$\bullet \geq 3 \mathrm{~mm}$ residual loss of $\mathrm{CAL}$ & 58 & 0 & \multirow{4}{*}{$\begin{array}{l}\text { Yes } \\
(p<0.001)\end{array}$} \\
\hline - 4-6 mm residual loss of CAL & 293 & $11^{\mathrm{d}}$ & \\
\hline - 7-9 mm residual loss of CAL & 166 & $34^{\mathrm{c}}$ & \\
\hline$\bullet \geq 10 \mathrm{~mm}$ residual loss of CAL & 56 & $62^{\mathrm{c}}$ & \\
\hline \multicolumn{4}{|l|}{ Tooth-related factors corrected } \\
\hline \multicolumn{4}{|l|}{ for disease status } \\
\hline \multicolumn{4}{|l|}{ Tooth type } \\
\hline - Single-rooted teeth & 435 & 14 & Yes \\
\hline - Multirooted teeth & 138 & 45 & $(p<0.001)$ \\
\hline \multicolumn{4}{|l|}{ Restorative treatment need ${ }^{\mathrm{b}}$} \\
\hline - Yes & 33 & 36 & No \\
\hline • No & 540 & 21 & $(p=0.260)$ \\
\hline \multicolumn{4}{|l|}{ Patient-related factors corrected } \\
\hline \multicolumn{4}{|l|}{ for disease status } \\
\hline \multicolumn{4}{|l|}{ Age (mean) } \\
\hline - Above & 288 & 19 & Yes \\
\hline • Under & 285 & 24 & $(p=0.007)$ \\
\hline \multicolumn{4}{|l|}{ Gender (mean) } \\
\hline - Above & 196 & 25 & \\
\hline - Under & 377 & 19 & $(p=0.860)$ \\
\hline \multicolumn{4}{|l|}{ Smoking } \\
\hline - Yes & 97 & 31 & No \\
\hline - No & 476 & 20 & $(p=0.161)$ \\
\hline \multicolumn{4}{|l|}{ Full-mouth plaque index (mean) } \\
\hline - Above & 320 & 20 & Yes \\
\hline - Under & 253 & 24 & $(p=0.046)$ \\
\hline \multicolumn{4}{|c|}{ Full-mouth bleeding on probing (mean) } \\
\hline - Above & 322 & 20 & No \\
\hline - Under & 251 & 23 & $(p=0.260)$ \\
\hline
\end{tabular}

CAL, clinical attachment level; $n$, number of teeth; PPD, probing pocket depth.

${ }^{\mathrm{a}}$ Mean proportion on a total of $\mathrm{n}$ teeth for which surgery was recommended by 15 clinicians; percentages shown.

${ }^{\mathrm{b}}$ Teeth in need of a crown.

${ }^{\mathrm{c}}$ Post-hoc test showing highly significant difference in comparison to all previous categories $(p<0.001)$.

${ }^{\mathrm{d}}$ Post-hoc test showing significant difference in comparison to previous category $(p=0.015)$.

prising, knowing that ample metaanalyses have indicated that deep sites benefit most from open flap debride- ment, whereas the benefit of surgery is less obvious at medium-to-deep sites (3-6). In addition, there was more 
disagreement for multirooted teeth than for single-rooted teeth. Apart from the presence of furcations in the multirooted teeth, which may increase complexity in decision making, it also has to be anticipated that the disease status was different between multiand single-rooted teeth. That is, the proportion of pockets exceeding $3 \mathrm{~mm}$ in probing pocket depth was considerably greater in multirooted teeth $(95 \%)$ than in single-rooted teeth $(58 \%)$, leaving more potential for disagreement for multirooted teeth.

Scrutinizing the results of this study revealed that experienced practitioners showed most variation in scoring, with a range from 13 to $50 \%$ in surgical recommendation. This observation suggests that clinical experience does not necessarily result in a uniform reasoning. Interestingly, substantial variation in periodontal diagnosis and treatment planning has recently been described among clinical instructors of one dental school (21). However, high interexaminer agreement of $90 \%$ for recommending surgery on a tooth-bytooth basis was previously reported when two experienced clinicians independently examined patients (22). The lack of accordance between this report and our results may be explained as follows: first, in the study by Loesche and coworkers (22), written criteria were used to assist the clinicians in making a decision, whereas in this study, no such criteria were provided in an attempt to simulate decision making as it actually occurs in clinical practice today. Second, disparities in the examiner's background should be acknowledged. That is, experienced clinicians in this study had been trained in different schools and worked in private practice. Consequently, there may have been variation in practice profile. In contrast, the two raters in the report by Loesche et al. (22) were associated with the same dental clinic. Finally, all decisions were based on data collected in our study without actually seeing the patients. This could be considered a drawback; yet, clinicians had access to all clinical data in order to make a rational decision. Furthermore, it would be technically impossible to have 23 patients clinically examined by 15 practitioners.

The results of the present study also showed that all clinicians associated with the dental school in Brussels scored in a very similar manner. In addition, the level of agreement seemed higher among the clinicians from the dental school in Ghent when compared to their agreement scores with practitioners from the two other groups. These findings indicate that clinicians linked to a training center shared a common treatment philosophy given by their instructors as to when periodontal surgery should be performed. Apparently, this philosophy differed substantially among both schools: clinicians linked to the dental school in Ghent advised more than twice as much surgery than their colleagues in Brussels (in general, 26\% vs. 11\%), despite a comparable number of years of clinical experience. If surgery was selected as the treatment of choice, clinicians in Brussels proportionally advised more surgery for multirooted teeth and for residual deep pockets. Interestingly, both philosophies seem to be supported by the existing literature: there is evidence indicating that chronic periodontitis can be succesfully controlled by means of a conservative strategy based on repetitive nonsurgical root debridement of residual pockets $(9,10,23)$, even though the need for subgingival re-instrumentation has recently been questioned (24). Alternatively, surgery may lead to fewer subjects requiring additional therapy in the long term (25). Moreover, the inability to provide intensive supportive care, especially because of a lack of dental hygienists in Belgium, may also justify a more drastic surgical approach, hereby engaging the most effective means for improving the periodontal status at deep sites.

Even though the clinical factors involved in making a surgical treatment decision have been described, there is still little standardization of the criteria upon which such a decision should be based. The results of this study indicate that practitioners interpret clinical data quite differently in their advice of surgery when clinical guidelines, in reference to surgical treatment, are not provided. The clinician's background seems to be a pivotal factor in selecting surgery as the treatment of choice, as clinicians linked to a training center showed more agreement in scoring with each other than with clinicians from another group. In contrast, experienced practitioners, who had all been trained in a different center, did not show more agreement in scoring with each other than with others. We wish to emphasize that it was not our objective to make a general judgement on surgical decision making by Belgian practitioners in training and private practice. However, the substantial variation in recommending surgical treatment by the convenience sample in this study is remarkable and calls for consensus statements describing clinical criteria assisting the practitioner in making a treatment decision. More standardization in this field may promote straightforward decision making, which is in the interest of the care provider and receiver. In addition, it is beneficial to a uniform periodontal education and may assist policy makers in implementing surgical periodontal therapy in healthcare insurance.

\section{Acknowledgements}

We express our sincere gratitude to all clinicians for their valuable contributions and to Prof. Dr L. Kaufman for sharing his expert knowledge on data analysis.

\section{References}

1. Baehni P, Giovannoli JL. Patient profile and decision-making in periodontal practice. Periodontol 2000 2004;36:27-34.

2. Loesche WJ, Taylor G, Giordano $\mathrm{J}$ et al. A logistic regression model for the decision to perform access surgery. $J$ Clin Periodontol 1997;24:171-179.

3. Antczak-Bouckoms A, Joshipura K, Burdick E, Tolloch JF. Meta-analysis of surgical versus non-surgical methods of treatment for periodontal disease. $J$ Clin Periodontol 1993;20:259-268.

4. Berkey CS, Antczak-Bouckoms A, Hoaglin DC, Mosteller F, Pihlstrom PL. Multiple-outcomes meta-analysis of treatments for periodontal disease. J Dent Res 1995;74:1030-1039.

5. Heitz-Mayfield LJA, Trombelli L, Heitz F, Needleman I, Moles D. A systematic 
review of the effect of surgical debridement versus non-surgical debridement for the treatment of chronic periodontitis. $J$ Clin Periodontol 2002;29:92-102.

6. Hung HC, Douglass CW. Meta-analysis of the effect of scaling and root planing, surgical treatment and antibiotic therapies on periodontal probing depth and attachment loss. J Clin Periodontol 2002; 29:975-986.

7. Lindhe J, Nyman S, Karring T. Scaling and root planing in shallow pockets. J Clin Periodontol 1982;9:415-418.

8. Nyman S, Lindhe J, Rosling B. Periodontal surgery in plaque-infected dentitions. J Clin Periodontol 1977;4:240249.

9. Axelsson P, Lindhe J. Effect of controlled oral hygiene procedures on caries and periodontal disease in adults. $J$ Clin Periodontol 1978;5:133-151.

10. Axelsson $\mathrm{P}$, Lindhe $\mathrm{J}$. The significance of maintenance care in the treatment of periodontal disease. J Clin Periodontol 1981;8:281-294.

11. Rosling B, Nyman S, Lindhe J, Jern B. The healing potential of the periodontal tissues following different techniques of periodontal surgery in plaque-free dentitions. A 2-year clinical study. $J$ Clin Periodontol 1976;3:233-255.
12. Harris RJ. Untreated periodontal disease: a follow-up on 30 cases. J Periodontol 2003;74:672-678.

13. Quigley GA, Hein JW. Comparative cleansing efficiency of manual and power brushing. J Am Dent Assoc 1962;65:26-29.

14. Mühlemann HR, Son S. Gingival sulcus bleeding - a leading symptom in initial gingivitis. Helv Odontol Acta 1971;15:107113.

15. Grembowski D, Milgrom P, Fiset L. Factors influencing dental decision-making. J Public Health Dent 1988;48:159-167.

16. Grembowski D, Milgrom P, Fiset L. Dental decision-making and variation in dentist service rates. Soc Sci Med 1991;32:287-294.

17. Warren JJ, Hand JS, Levy SM, Kirchner HL. Factors related to decisions to extract or retain at-risk teeth. $J$ Public Health Dent 2000;60:39-42.

18. Brennan DS, Spencer AJ. Factors influencing choice of dental treatment by private general practitioners. Int $J$ Behav Med 2002;9:94-110.

19. Mordohai D, Reshad M, Jivray SA. To extract or not to extract? Factors that affect individual tooth prognosis. $J$ Calif Dent Assoc 2005;33:319-328.

20. Patel AM, Richards PS, Wang HL, Inglehart MR. Surgical or non-surgical periodontal treatment: factors affecting patient decision-making. $J$ Periodontol 2006;77:678-683.

21. Lanning SK, Pelok SD, Williams BC et al. Variation in periodontal diagnosis and treatment planning among clinical instructors. J Dent Educ 2005;69:325-337.

22. Loesche WJ, Giordano J, Soehren S et al. Nonsurgical treatment of patients with periodontal disease. Oral Surg Oral Med Oral Pathol Oral Radiol Endod 1996;81:533-543.

23. Rosling B, Serino G, Hellstrom MK, Socransky SS, Lindhe J. Longitudinal periodontal tissue alterations during supportive therapy. Findings from subjects with normal and high susceptibility to periodontal disease. J Clin Periodontol 2001;28:241-249.

24. Heasman PA, McCracken GI, Steen N. Supportive periodontal care: the effect of periodic subgingival debridement compared with supragingival prophylaxis with respect to clinical outcomes. J Clin Periodontol 2002;29:163-172.

25. Serino G, Rosling B, Ramberg $P$, Socransky SS, Lindhe J. Initial outcome and long-term effect of surgical and nonsurgical treatment of advanced periodontal disease. $J$ Clin Periodontol 2001;28:910-916. 